\title{
The Sphaleron Rate through the Electroweak Cross-over
}

\author{
Michela D'Onofrio, ${ }^{a}$ Kari Rummukainen ${ }^{a}$ and Anders Tranberg $^{b}$ \\ ${ }^{a}$ Department of Physics and Helsinki Institute of Physics, \\ University of Helsinki, P.O.Box 64, FIN-00014 Helsinki, Finland \\ ${ }^{b}$ Niels Bohr International Academy, Blegdamsvej 17, 2100 Copenhagen, Denmark \\ E-mail: michela.donofrio@helsinki.fi, kari.rummukainen@helsinki.fi, \\ anders.tranberg@nbi.fi
}

\begin{abstract}
Using lattice simulations, we measure the sphaleron rate in the Standard Model as a function of temperature through the electroweak cross-over, for the Higgs masses $m_{H}=115$ and $m_{H}=160 \mathrm{GeV}$. We pay special attention to the shutting off of the baryon rate as the temperature is lowered. This quantity enters computations of Baryogenesis via Leptogenesis, where non-zero lepton number is converted into non-zero baryon number by equilibrium sphaleron transitions. Combining existing numerical methods applicable in the symmetric and broken electroweak phases, we find the temperature dependence of the sphaleron rate at very high temperature, through the electroweak cross-over transition, and deep into the broken phase.
\end{abstract}

KEYwords: Baryogenesis, Sphaleron rate, Electroweak physics, Lattice simulations 


\section{Contents}

1 Introduction $\quad 1$

1.1 Calculations of the sphaleron rate 2

1.2 The sphaleron rate and Baryogenesis 4

2 SU(2)-Higgs model on the lattice 4

2.1 Dimensional reduction 5

2.2 Lattice-continuum relations 6

$\begin{array}{lll}2.3 & \text { Real-time evolution } & 7\end{array}$

3 Measuring the sphaleron rate $\quad 8$

3.1 Sphaleron rate in the symmetric phase $\quad 9$

3.2 Sphaleron rate in the broken phase: multicanonical method 9

3.3 Sphaleron rate in the broken phase: dynamical prefactor 13

4 Results $\quad \mathbf{1 5}$

$\begin{array}{lll}4.1 & \text { Higgs field } v(T) & 15\end{array}$

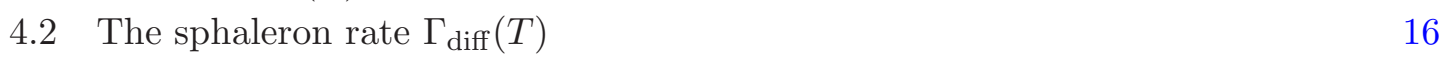

$\begin{array}{lll}5 & \text { A sample Leptogenesis calculation } & 19\end{array}$

6 Conclusion 21

\section{Introduction}

As a result of a quantum anomaly, baryon number $B$ and the lepton numbers $L_{i}$ are not strictly conserved charges in the Standard Model. As the Chern-Simons number $N_{\mathrm{CS}}$ of the gauge field evolves in time, because only left-handed fermions are coupled to the $\mathrm{SU}(2)$ gauge field, the baryon and lepton numbers $B$ and $L_{i}$ change according to the relation (see for instance $[1,2]$ )

$$
\frac{1}{n_{G}}[B(t)-B(0)]=L_{i}(t)-L_{i}(0)=N_{\mathrm{CS}}(t)-N_{\mathrm{CS}}(0)=\frac{1}{32 \pi^{2}} \int_{0}^{t} d t \int d^{3} x \operatorname{Tr} F^{\mu \nu} \tilde{F}_{\mu \nu},
$$

where $F^{\mu \nu}$ is the field strength tensor of the $\mathrm{SU}(2)$ gauge field and $n_{G}=3$ is the number of fermion generations in the Standard Model.

In addition, the electroweak sector of the Standard Model has an infinite set of "pure gauge" degenerate vacua, corresponding to integer values $N_{\mathrm{CS}}$. The question of baryon and lepton number violation in the Standard Model therefore becomes a question of whether dynamics allow transitions from one vacuum to another. 
At zero temperature transitions occur via instantons [3], and the rate is minute, negligible even on cosmological scales. However at finite temperature, thermal fluctuations can lead to spontaneous transitions, controlled in equilibrium by a diffusion (or sphaleron) rate $\Gamma_{\text {diff }}$,

$$
\Gamma_{\text {diff }}(T)=\lim _{V, t \rightarrow \infty} \frac{\left\langle\left[N_{\mathrm{CS}}(t)-N_{\mathrm{CS}}(0)\right]^{2}\right\rangle}{V t} .
$$

At zero temperature, the Standard Model Higgs field acquires a vacuum expectation value $\langle|\phi|\rangle=v / \sqrt{2}$, with $v=246 \mathrm{GeV}$. In contrast, at high temperature $\langle|\phi|\rangle \approx 0$, and there is a "symmetry breaking" transition between the two phases ("symmetric" and "broken"). Note that, because of gauge symmetry and Elitzur's theorem, $\langle\phi\rangle$ is always zero, whereas $\langle|\phi|\rangle$ is always non-zero (the value depends on the renormalization scheme, though). Thus, we do not have an exact local order parameter which would vanish in the symmetric phase and be non-vanishing in the broken phase. Indeed, at low Higgs masses $\left(m_{H} \lesssim 72 \mathrm{GeV}\right)$, the Standard Model phase transition is an actual first-order phase transition, but at experimentally allowed values for the mass the electroweak transition in the Minimal Standard Model is a continuous cross-over [4-7]. Nevertheless, we shall use the labels "symmetric" and "broken" phases to describe the states above and below the cross-over temperature.

In the symmetric phase the sphaleron rate is unsuppressed and is proportional to $T^{4}$. In the broken phase the energy barrier between the topological sectors grows as $\langle|\phi|\rangle$ increases, and parametrically the rate is

$$
\frac{\Gamma_{\text {diff }}^{\mathrm{brok}}(T)}{T^{4}}=\kappa_{\mathrm{brok}} \alpha_{w}^{4} e^{-\frac{E_{\mathrm{sph}}}{T}},
$$

where $E_{\mathrm{sph}}$ is the sphaleron energy (energy barrier) and $\kappa_{\text {brok }}$ is a numerical coefficient [8]. In the physical cross-over case it is of interest to know how the rate "shuts off" as temperature decreases, including the precise determination of the rate in the exponentially suppressed temperature range. This is important e.g. for Leptogenesis scenarios, as discussed in Sect. 5 .

\subsection{Calculations of the sphaleron rate}

The sphaleron rate has been computed extensively using lattice simulations. For pure $\mathrm{SU}(2)$ gauge theory, the quantum diffusion rate is approximately recovered in the classical dynamics of a dimensionally reduced effective theory [9-12], taking into account the proper conversion factors $[13,14]$. The calculation was improved by including the effect of hard thermal loops $[15,16]$, and the magnitude was finally settled using the Langevin dynamics of another effective theory [17, 18], again using a conversion prescription [18-20].

The rate in the electroweak theory (i.e. including the Higgs scalar) was computed in the symmetric phase at $m_{H} \simeq 34 \mathrm{GeV}$ in [11], and for $m_{H} \simeq m_{W}$ in [21] in approximate agreement with the pure-gauge result. The most recent simulations [22] combine all previous methods, extends the effective theory of [17] to include the Higgs field, and finds that at $m_{H} \simeq 44 \mathrm{GeV}[22]$,

$$
\frac{\Gamma_{\text {diff }}^{\text {sym }}}{T^{4}}=(8.24 \pm 0.10)\left(\frac{g^{2} T^{2}}{m_{D}^{2}}\right)\left(\ln \left(\frac{m_{D}^{2}}{g^{2} T}\right)+C\right) \alpha_{w}^{5},
$$


with

$$
m_{D}^{2}=\frac{11}{6} g^{2} T^{2}, \quad C=3.041, \quad \alpha_{w}=\frac{g^{2}}{4 \pi} .
$$

In the broken phase, the rate is less well known. The straightforward numerical methods of $[11,21]$ are not able to cope with the large suppression of the rate, a problem resolved by Moore using multicanonical methods [23, 24]. In these papers the low Higgs mass region, where the phase transition is strongly first order, was considered. The latter paper finds that at the transition temperature $T_{c}$ the broken phase rate is

$$
\frac{\Gamma_{\text {diff }}^{\mathrm{brok}}\left(T=T_{c}\right)}{T_{c}^{4}}=\exp \left[-R\left(\frac{m_{H}}{\mathrm{GeV}}\right)\right]
$$

with

$$
R(49.2)=24.7 \pm 0.4, \quad R(44.8)=28.3 \pm 0.4, \quad R(41.2)=31.2 \pm 0.6 .
$$

In this case the strong suppression in the broken phase results in an effectively instantaneous shut-off of sphaleron processes during the strong first-order phase transition. In the crossover regime, one would expect a gradual shut-off as the Higgs field expectation value increases.

In [25] the sphaleron rate was calculated in the electroweak cross-over region, around $m_{H}=120 \mathrm{GeV}$, from high-temperature into the low-temperature phase, using classical dynamics. Shortly afterwards [22], a similar calculation was performed at $m_{H}=130 \mathrm{GeV}$, but using the effective dynamics of [17]. In both cases a rather rapid shut-off of sphaleron diffusion through the cross-over was reported. However, because no multicanonical methods were used, the exponentially suppressed tail was not resolved in detail. The aim of this paper is to improve on these calculations, and pin down the sphaleron rate through the cross-over, also in the domain of exponential suppression.

Some time ago [26], known calculations of the sphaleron rate were collected and extrapolated to the cross-over region. The conclusion was that within a range

$$
100 \mathrm{GeV} \leq m_{H} \leq 200 \mathrm{GeV},
$$

and for a range of $T$ so that

$$
-\ln \left[\Gamma_{\text {diff }}(T) / T^{4}\right] \simeq 30-50,
$$

we have

$$
-\ln \left[\frac{\Gamma_{\text {diff }}}{T^{4}}\right] \simeq \sum_{i, j} c_{i j}\left(\frac{m_{H}-150 \mathrm{GeV}}{10 \mathrm{GeV}}\right)^{i}\left(\frac{T-150 \mathrm{GeV}}{10 \mathrm{GeV}}\right)^{j},
$$

with $c_{00}=39.6, c_{10}=3.52, c_{01}=-7.09, c_{20}=-0.376, c_{11}=0.421, c_{02}=0.17$. We will use this result for guidance and establish its range of validity.

In this work we study the sphaleron rate at Higgs mass values $m_{H}=115 \mathrm{GeV}$ and $m_{H}=160 \mathrm{GeV}^{1}$, with special attention paid at the rate deep in the broken phase.

\footnotetext{
${ }^{1}$ These Higgs mass values were within the experimentally allowed range when the simulations were started [27].
} 


\subsection{The sphaleron rate and Baryogenesis}

In Electroweak Baryogenesis [1, 2], bubble nucleation in a first-order electroweak phase transition divides space into regions of different electroweak phases. Inside the bubbles is the broken phase where the sphaleron rate is very small. Outside the bubbles is the symmetric phase, where the rate is large. CP-violating interactions between the fermions in the plasma and the advancing bubble walls generate a net chiral fermion current, which is then transformed, by sphaleron processes, into a baryon and lepton asymmetry [28]. A first-order phase transition is not realized in the Minimal Standard Model, but the Baryogenesis scenario may be relevant for extensions such as the 2-Higgs Doublet Model and Supersymmetric Standard Models, in which case a reliable calculation of the sphaleron rate in both phases is important.

In Baryogenesis via Leptogenesis [29, 30], a lepton asymmetry is assumed to originate from some separate process, represented here by a time-dependent source $f_{i}(t)$ which may or may not be active at the electroweak scale. Sphaleron transitions equilibrate the system, so that the lepton asymmetry is transformed into net $L_{i}$ and $B$. Following [26], the equations controlling this equilibration read

$$
\begin{aligned}
& \dot{B}(t)=-\gamma(t)\left[B(t)+\eta(t) \sum_{i=0}^{n_{G}} L_{i}(t)\right] \\
& \dot{L}_{i}(t)=-\frac{\gamma(t)}{n_{G}}\left[B(t)+\eta(t) \sum_{i=0}^{n_{G}} L_{i}(t)\right]+f_{i}(t),
\end{aligned}
$$

where $T=T(t), \gamma(t)=\gamma\left[\Gamma_{\text {diff }}(T), v(T)\right], \eta(t)=\eta[v(T)]$ and $n_{G}=3 . v(T)$ is the expectation value of the Higgs field, taken to be $v(T) \simeq \sqrt{\left\langle\phi^{\dagger} \phi\right\rangle}$.

In this paper, we will calculate $\Gamma_{\text {diff }}(T)$ and $v(T)$, in the Minimal Standard Model at experimentally allowed Higgs masses, i.e. in the regime where the electroweak transition is an equilibrium cross-over.

In Section 2 we briefly explain how to treat the dynamics of the Standard Model at finite temperature as an effective 3-dimensional SU(2)-Higgs theory. Section 3 we describe the numerical lattice Monte-Carlo methods employed here, in the high- and lowtemperature regimes, respectively. In Section 4 we present our results for $\Gamma_{\text {diff }}(T)$ and $v(T)$. In Section 5 we solve (5.2) for some simple cases to assess the impact of including the correct sphaleron rate rather than assuming an instantaneous shut-off. We conclude in Section 6 .

\section{$2 \mathrm{SU}(2)$-Higgs model on the lattice}

The sphalerons are non-perturbative field configurations and thus we need to use nonperturbative lattice simulations in order to calculate the rate reliably. Furthermore, because of the infrared problem in the thermodynamics of the non-Abelian Yang-Mills theory, the modes with momenta $k \leq g^{2} T$ are non-perturbative. This means that the perturbative expansion becomes impossible beyond some loop order [31]. 


\subsection{Dimensional reduction}

The full four-dimensional Standard Model with chirally coupled fermions is too unwieldy to simulate on the lattice. However, for static (time-independent) thermodynamics we can use the fact that the weak coupling constant is small, and apply perturbation theory only to modes which can be reliably treated with perturbative methods: that is, to modes with momentum $k>g^{2} T$, where $g^{2}$ is the weak gauge coupling. This procedure is called dimensional reduction, because it results in a three-dimensional effective theory for the soft $\left(g^{2} T\right)$ modes. The effective theory is purely bosonic, and it fully includes the essential non-perturbative physics. The detailed description how this is performed can be found in ref. [32-34]; for earlier and related work, see [35-40].

The perturbative derivation of the effective theory is based on the hierarchy between the hard $(k \gtrsim T)$, electric $(k \sim g T)$ and magnetic $\left(k \sim g^{2} T\right)$ scales on an Euclidean finitetemperature path integral. In the first stage we integrate over the hard scales, obtaining an effective theory of scales $k \lesssim g T$. Because the Matsubara frequencies for the bosonic and fermionic field modes are $k_{0}^{\text {boson }}=2 \pi n T$ and $k_{0}^{\text {fermion }}=\pi(2 n+1) T, n \in Z$ all fermionic modes and non-static $\left(k_{0} \neq 0\right)$ bosonic modes are of order $T$. Thus, the effective theory is purely bosonic and three-dimensional. Concretely, the actual "integration" is done by writing down a general renormalizable effective theory and matching the perturbatively computed two-, three- and four-point functions in the effective theory and in the original four-dimensional theory, thus fixing the parameters of the effective theory.

The effective theory can be further simplified by integrating over scales $g T$, which gives us the three-dimensional SU(2) gauge theory coupled to a scalar (Higgs) field: ${ }^{2}$

$$
S=\int d^{3} x\left(\frac{1}{4} F_{i j}^{a} F_{i j}^{a}+\left(D_{i} \phi\right)^{\dagger}\left(D_{i} \phi\right)+m_{3}^{2} \phi^{\dagger} \phi+\lambda_{3}\left(\phi^{\dagger} \phi\right)^{2}\right) .
$$

The coupling constants of the theory are $g_{3}^{2} \sim g^{2} T, m_{3}^{2}$ and $\lambda_{3}$. These depend on temperature and the parameters of the Standard Model; the full expressions are given in ref. [32]. It is customary to use dimensionless quantities $x$ and $y$ and express the set of the parameters of the effective theory as

$$
g_{3}^{2}, \quad x=\frac{\lambda_{3}}{g_{3}^{2}}, \quad y=\frac{m_{3}^{2}}{g_{3}^{4}} .
$$

Here the dimensionful parameter $g_{3}^{2}$ gives the scale and the physics is completely determined by the values of $x$ and $y$.

The values of $x$ and $y$ for the Higgs masses used in this work, $m_{H}=115 \mathrm{GeV}$ and $m_{H}=160 \mathrm{GeV}$, are shown in Fig. 1 over the relevant temperature range. The other significant Standard Model parameters which influence the values of $x$ and $y$ are the Fermi coupling $G_{F}=1.16639 \times 10^{-5}, m_{Z}=91.1876 \mathrm{GeV}, m_{W}=80.425 \mathrm{GeV}, m_{t}=174.3 \mathrm{GeV}$ and $\alpha_{S}\left(m_{W}\right)=0.1187$.

\footnotetext{
${ }^{2}$ The hypercharge $\mathrm{U}(1)$ and gluon $\mathrm{SU}(3)$ gauge fields in principle survive the dimensional reduction. However, the gluons do not couple to the weak gauge and Higgs fields and only modify the parameters in Eq. (2.1) through loop corrections. The U(1) field is omitted here because its effect on the transition is numerically small [4].
} 

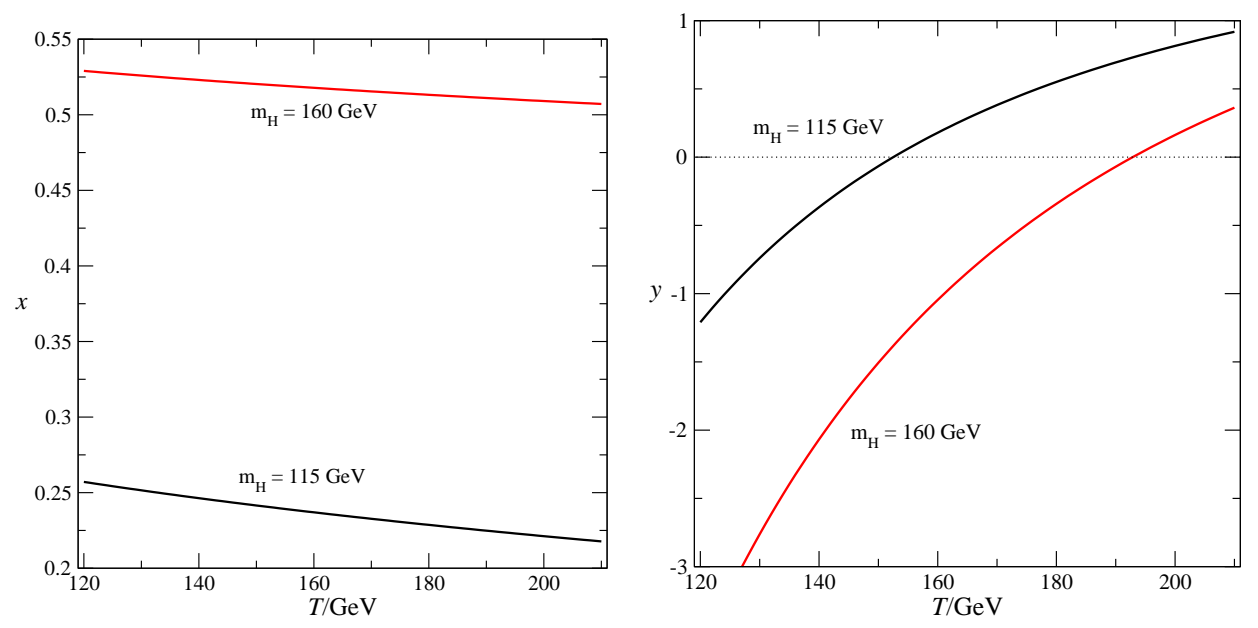

Figure 1. The values of $x$ (left) and $y$ (right) for $m_{H}=115$ and $160 \mathrm{GeV}$ in the temperature range of interest.

\section{$2.2 \quad$ Lattice-continuum relations}

At this point we have built a continuum 3D theory from the $4 \mathrm{D}$ fundamental one. Now we have to put it on the lattice. We use here the common lattice discretization of the action [5]

$$
\begin{aligned}
S_{\text {Lat }} & =\beta_{G} \sum_{x} \sum_{i<j}\left(1-\frac{1}{2} \operatorname{Tr}\left[P_{i j}\right]\right)-\beta_{H} \sum_{x} \sum_{i} \frac{1}{2} \operatorname{Tr}\left[\Phi^{\dagger}(x) U_{i}(x) \Phi(x+\hat{i})\right]+ \\
& +\sum_{x} \frac{1}{2} \operatorname{Tr}\left[\Phi^{\dagger}(x) \Phi(x)\right]+\beta_{R} \sum_{x}\left[\frac{1}{2} \operatorname{Tr}\left[\Phi^{\dagger}(x) \Phi(x)\right]-1\right]^{2}
\end{aligned}
$$

which is constructed only with gauge-invariant terms. Here $U_{i}(x)$ is the $\mathrm{SU}(2)$ gauge link variable, $P_{i j}$ is the standard $i j$-plane plaquette constructed from the link variables, and the lattice Higgs field is naively scaled from the continuum field with $(1 / 8) \beta_{G} \beta_{H} \Phi^{\dagger} \Phi=\phi^{\dagger} \phi / g_{3}^{2}$. The parameters $\beta_{G}, \beta_{H}$ and $\beta_{R}$ are related to the parameters $g_{3}^{2} a, x$ and $y$ by

$$
\begin{aligned}
\beta_{G}= & \frac{4}{g_{3}^{2} a}, \\
x= & \frac{1}{4} \lambda_{3} a \beta_{G}=\frac{\beta_{R} \beta_{G}}{\beta_{H}^{2}}, \\
y= & \frac{\beta_{G}^{2}}{8}\left(\frac{1}{\beta_{H}}-3-\frac{2 x \beta_{H}}{\beta_{G}}\right)+\frac{3 \Sigma \beta_{G}}{32 \pi}(1+4 x)+\frac{1}{16 \pi^{2}} . \\
& \cdot\left[\left(\frac{51}{16}+9 x-12 x^{2}\right)\left(\ln \frac{3 \beta_{G}}{2}+\zeta\right)+5.0+5.2 x\right] .
\end{aligned}
$$

Here $\Sigma=3.1759115$ and $\zeta=0.08849$ and the other numerical constants appearing in (2.6) are specific for the $\mathrm{SU}(2)+$ Higgs model and are computed in [41].

We note here that because the action is superrenormalizable, only the mass term $(y)$ gets renormalized. The continuum limit is taken using equations (2.4)-(2.6) by letting 
$\beta_{G} \rightarrow \infty$ while keeping $x, y$ and $g_{3}^{2}$ constant. The counterterms in Eq. (2.6) remove the linear and logarithmic divergences in the lattice spacing $a$.

We actually implement the following simple improvement to equations (2.4)-(2.6), which cancels part of the $O(a)$ corrections [42]: in the lattice action (2.3) and equations (2.5)-(2.6) we substitute

$$
\beta_{G} \rightarrow \beta_{G, \text { improved }}=4 /\left(g_{3}^{2} a\right)+0.6674
$$

which makes the gauge part of the action $O(a)$ accurate. In fact, $(2.5)$ can also be $O(a)$ improved [42], but since the full $O(a)$ correction to (2.6) is not known we do not implement it here. In what follows we consistently use the identity $\beta_{G}=4 /\left(g_{3}^{2} a\right)$.

The gauge invariant Higgs field expectation value $\left\langle\Phi^{\dagger} \Phi\right\rangle$ has linear and logarithmic UV divergences. Subtracting the divergences from the lattice expectation value, we obtain the continuum quantity $\left\langle\phi^{\dagger} \phi\right\rangle$ as [5] using

$$
\frac{\left\langle\phi^{\dagger} \phi\right\rangle}{g_{3}^{2}}=\frac{1}{8} \beta_{G} \beta_{H}\left(\left\langle\Phi^{\dagger} \Phi\right\rangle-\frac{\Sigma}{\pi \beta_{H}}\right)-\frac{3}{(4 \pi)^{2}}\left(\log \frac{3 \beta_{G} g_{3}^{2}}{2 g_{3}^{2}}+\zeta+\frac{1}{4} \Sigma^{2}-\delta\right)+O\left(\frac{1}{\beta_{G}}\right)
$$

where $\zeta+\frac{1}{4} \Sigma^{2}-\delta \approx 0.6678$

\subsection{Real-time evolution}

The effective theory in Eq. (2.1) is well understood and has been very successfully used in studies of static thermodynamical quantities of hot electroweak physics. As such, it does not describe dynamical phenomena, which include sphaleron transitions. It is possible to take the theory in Eq. (2.1) and use the classical equations of motion to describe the time evolution of the fields, as was done in the early studies of the sphaleron rate [11, 21, 23]. However, it has been shown that the classical theory contains divergent UV contributions to the gauge field dynamics, and the results are cut-off dependent [43]. Hence, technically the infrared gauge field dynamics of the classical theory do not exist. The physical origin of the problem is that the Landau damping of the transverse gauge fields in the classical theory is UV divergent, and the theory does not have a physical continuum limit.

These problems can be ameliorated by studying classical theory with hard thermal loop (HTL) effects included [44]. This leads to complicated and expensive numerical implementations $[15,16]$. However, as first demonstrated by Bödeker [17], the physical damping makes the dynamics of the infrared gauge field modes (modes with $k \gtrsim g^{2} T$ ) to be fully overdamped. Then, at leading order in $1 / \ln (1 / g)$ the evolution of these modes is described with simple Langevin dynamics (in $A_{0}=0$ gauge, and identifying $H / T=S$ in (2.1)) [20]:

$$
\partial_{t} A_{i}=-\sigma_{\mathrm{el}}^{-1} \frac{\partial H}{\partial A_{i}}+\xi_{i}^{a}
$$

where $\sigma_{\mathrm{el}}$ is the non-Abelian "color" conductivity,

$$
\sigma_{\mathrm{el}}^{-1}=\frac{3 \gamma}{m_{D}^{2}}, \quad \gamma=\frac{2 g^{2} T}{4 \pi}\left(\ln \frac{m_{D}}{\gamma}+3.041\right)
$$


and again $m_{D}^{2}=(11 / 6) g^{2} T^{2}$ in the standard model. $\xi$ is a random Gaussian noise with

$$
\left\langle\xi_{i}^{a}(\mathbf{x}, t) \xi_{j}^{b}\left(\mathbf{x}^{\prime}, t^{\prime}\right)\right\rangle=2 \sigma_{\mathrm{el}} T \delta_{i j} \delta^{a b} \delta\left(\mathbf{x}-\mathbf{x}^{\prime}\right) \delta\left(t-t^{\prime}\right) .
$$

The Higgs field has parametrically much less damping. Hence, it can also be evolved with a Langevin equation, but with a much faster rate of evolution. To this accuracy we can take it to be infinitely fast in comparison with the gauge field evolution [22]. Iterating, we can solve for $\gamma=0.66361688 g^{2} T$.

In principle the Langevin evolution is straightforward to implement on the lattice. However, it is unnecessarily slow: we can substitute it with any dissipative update, as long as the relation between the evolution rates is known. Thus, it is much more efficient to use random-order heat-bath update algorithm for the $\mathrm{SU}(2)$ gauge fields $[18,45]$. Now $n$ full heat-bath update sweeps through the lattice correspond to the real-time step

$$
\Delta t=\frac{1}{4} \sigma_{\mathrm{el}} a^{2} n .
$$

We note that this relation is valid for "unimproved" single-plaquette Wilson gauge and random-order heat bath; for other choices the relation would differ. The Higgs field is updated with a mixture of heat bath and overrelaxation much more frequently than the gauge field [45].

\section{Measuring the sphaleron rate}

The evolution of the Chern-Simons number $N_{\mathrm{CS}}$ over a time interval $\left(t_{0}, t\right)$ can be defined using lattice electric and magnetic fields:

$$
\delta N_{\mathrm{CS}}(t) \equiv N_{\mathrm{CS}}(t)-N_{\mathrm{CS}}\left(t_{0}\right)=\frac{g^{2}}{8 \pi^{2}} \int_{t_{0}}^{t} d t^{\prime} \int d^{3} x E_{i}^{a} B_{i}^{a} .
$$

Unfortunately the topology on the lattice is not well defined, and using naive lattice scale $E$ and $B$ fields the right-hand side of equation (3.1) contains ultraviolet noise. This gives unphysical diffusion not connected with the sphaleron rate. The method of calibrated cooling [23] (see also [11]), offers a way out of the problem. It is based on the fact that at small enough lattice spacing sphalerons are large in lattice units, with a dominant length scale of order $1 /\left(g^{2} T\right) .{ }^{3}$ By applying a pre-determined amount of cooling (Langevin evolution without the noise) to the lattice gauge fields, the ultraviolet noise is eliminated, without compromising the long-distance topology of the configuration. At this point it is possible to evaluate the integral in (3.1) with only small errors. Cumulative residual errors are eliminated by periodically cooling all the way down to a vacuum configuration and correcting for deviation from integer values of $\delta N_{C S}$ between two vacua. This is schematically described in Figure 2. By adjusting the cooling parameters so that these deviations from integers always remain much smaller than unity, we also ensure that the cooling is sufficient to keep the measurement topological.

\footnotetext{
${ }^{3}$ In ref. [12] the size of the sphaleron was estimated to be of order $5 /\left(g^{2} T\right)$ in the pure $\mathrm{SU}(2)$ gauge theory.
} 


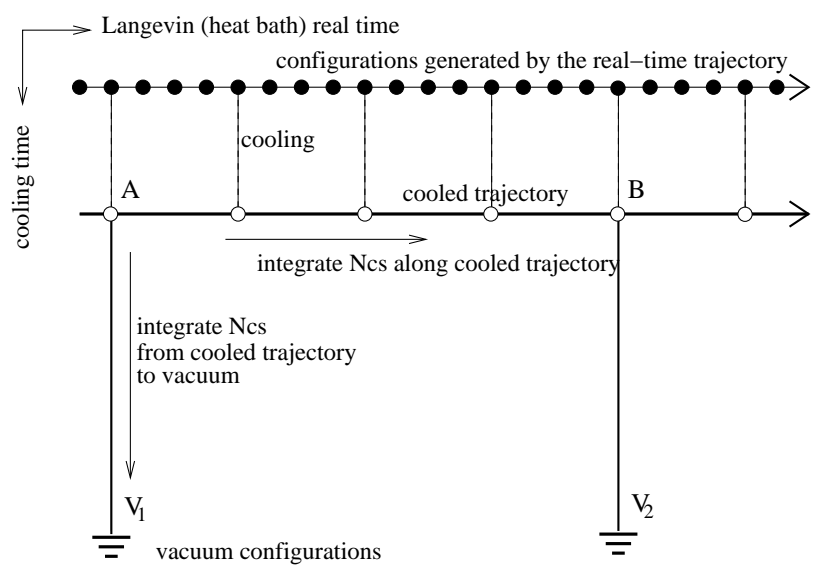

Figure 2. Measurement of the Chern-Simons number evolution [23]. The solid circles show the configurations generated by the real-time evolution using the Langevin/heat-bath method. At fixed intervals, the configurations are cooled by the same amount in order to construct a cooled trajectory, where the UV noise is almost completely eliminated, allowing to calculate $\delta N_{\mathrm{CS}}$ from (3.1). The cooling from vacuum to vacuum works as a test for residual errors: $\delta N_{\mathrm{CS}}$ must then be close to an integer, the deviations from which are subtracted, thus avoiding the accumulation of errors.

Cooling the original gauge fields close to the vacuum is computationally very expensive. The procedure is dramatically accelerated by blocking the lattice gauge fields by a factor of two after the UV noise has been sufficiently eliminated; this is repeated a couple of times until a minimum lattice size has been reached. For details, we refer to [23].

\subsection{Sphaleron rate in the symmetric phase}

We calculate the sphaleron rate using two different, and complementary, methods. We start at high temperatures, above the cross-over, and go through the whole cross-over range into the "broken phase" by uniformly decreasing the temperature. At high temperatures we use standard canonical Monte Carlo sampling. As the potential barrier between consecutive Chern-Simons numbers is low, the probability distribution over Chern-Simons number is approximately flat. As we decrease the temperature, the sphaleron rate becomes exponentially suppressed and the canonical real-time method is too inefficient to resolve the slow rate. At this point we begin using multicanonical simulations.

As an example, the evolution of the Chern-Simons number at $m_{H}=115 \mathrm{GeV}$ and with $T=152 \mathrm{GeV}$ (symmetric phase), $145 \mathrm{GeV}$ (cross-over region) and $140 \mathrm{GeV}$ (broken phase) is shown in Figure 3. In the symmetric phase the transitions are unsuppressed and it is straightforward to measure the diffusion rate. Around the cross-over temperature the probability distribution of $\Delta N_{\mathrm{CS}}$ becomes peaked around integer values and the transitions between these values become rapidly more suppressed. Finally, deep in the broken phase the rate goes down until we are not able to measure it with the real-time evolution method.

\subsection{Sphaleron rate in the broken phase: multicanonical method}

At low temperatures where the sphaleron rate is strongly suppressed, this can be measured using a multicanonical method similar to the one used in [23]. The calculation consists of 

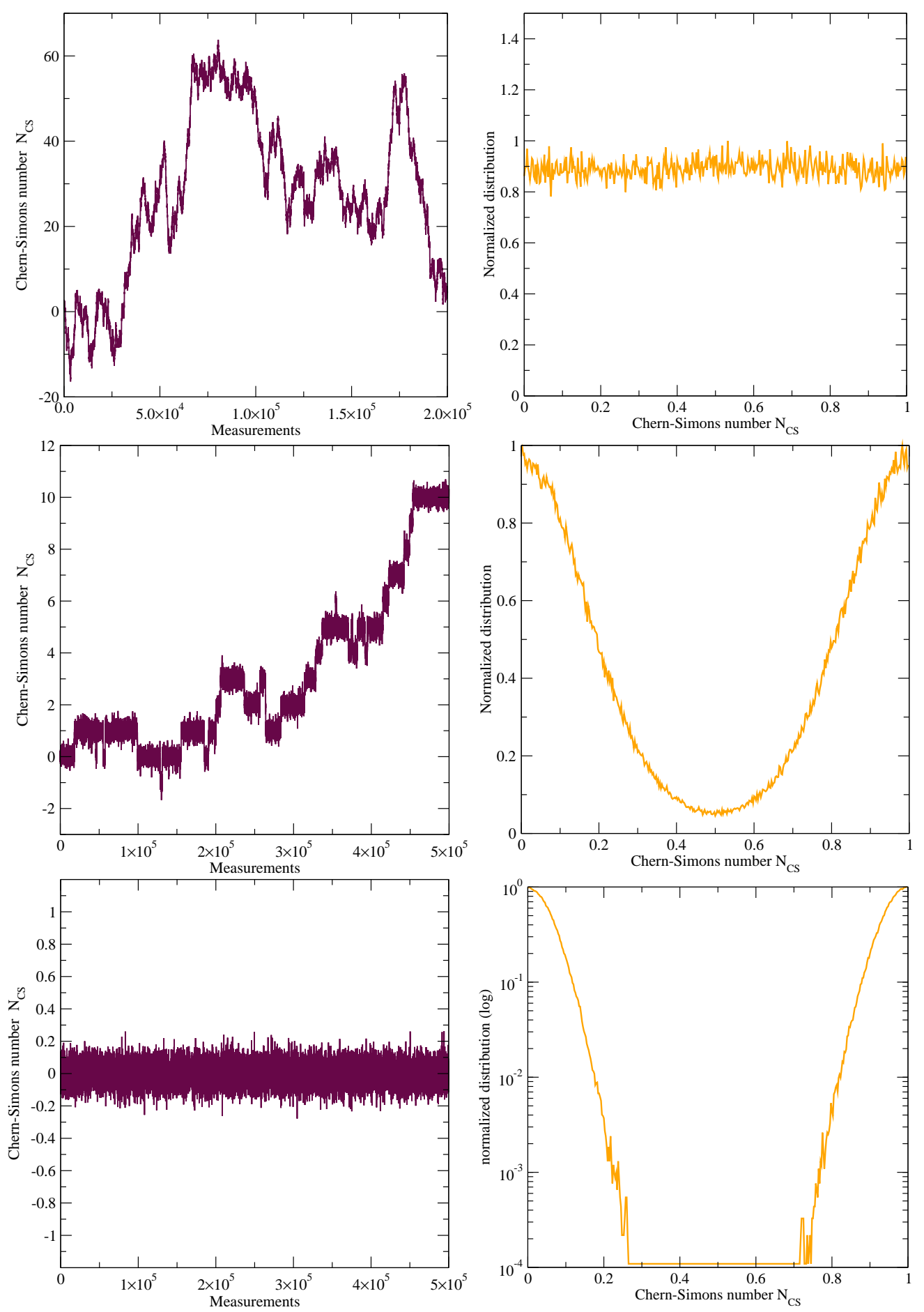

Figure 3. $N_{\mathrm{CS}}$ from a heat-bath trajectory (left), and the resulting probability distribution (right), folded into the interval $[0,1]$, at $m_{H}=115 \mathrm{GeV}$ and $T=152$ (top), 145 (middle) and $140 \mathrm{GeV}$ (bottom). At high temperature, in the symmetric phase, the sphaleron transitions are unsuppressed, whereas at low $T$ the transitions are so strongly suppressed that they do not happen in canonical simulations. 

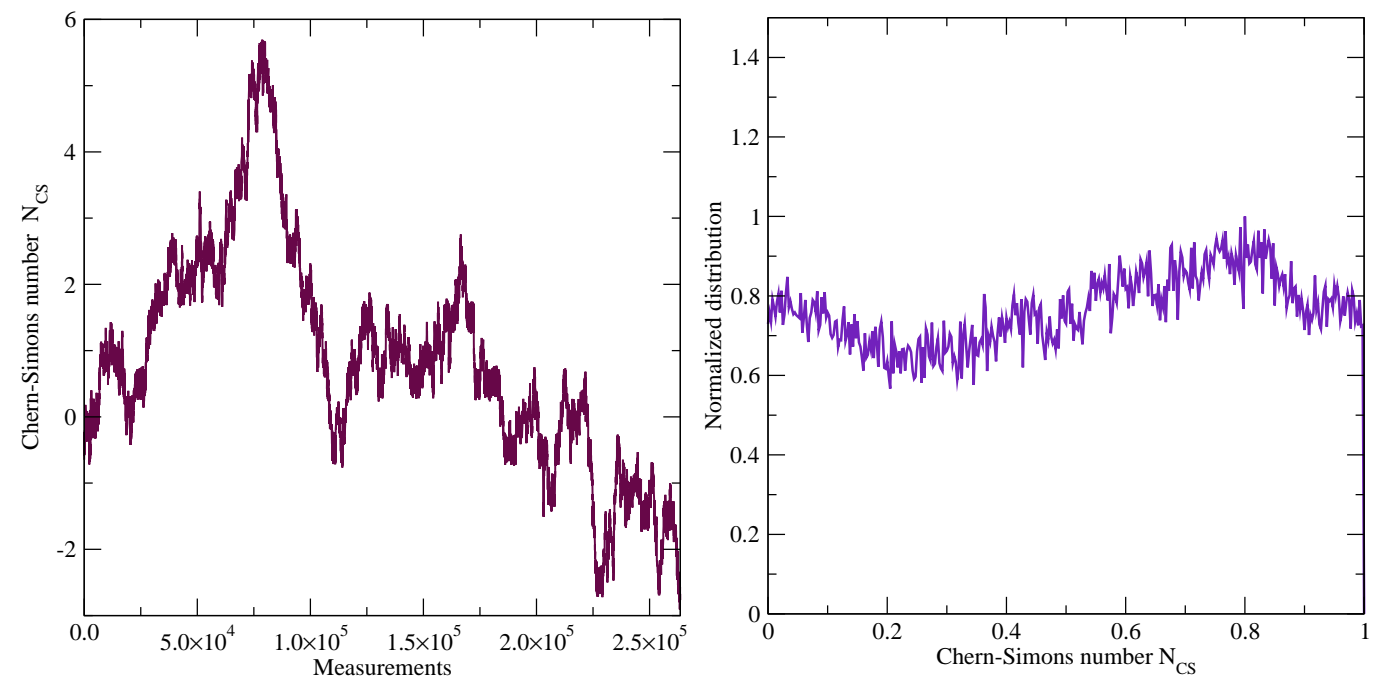

Figure 4. A heat-bath trajectory for $N_{\mathrm{CS}}$, still for $m_{H}=115 \mathrm{GeV}$ and $T=140$ but now with multicanonical simulations (left), and the corresponding multicanonical probability distribution $P_{\text {muca }}($ right).
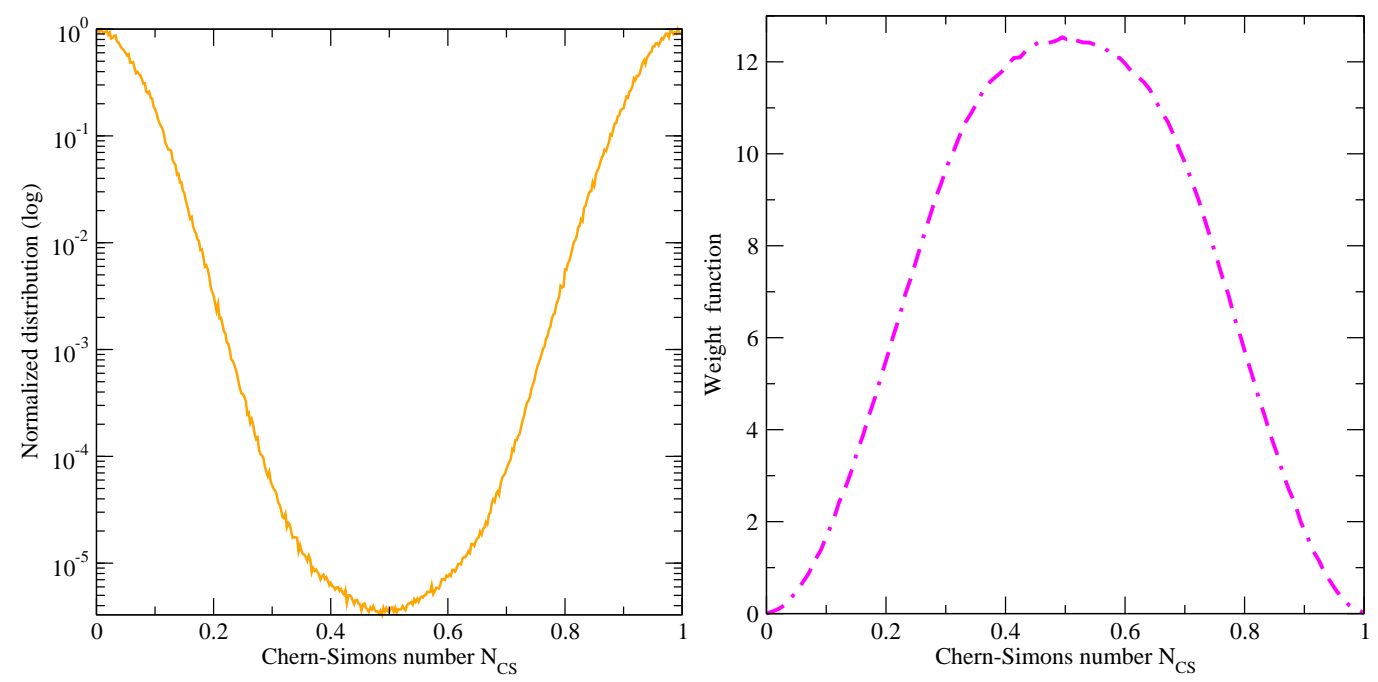

Figure 5. The physical distribution $P_{\text {can }}$ (left), after reweighting the result in figure 3.2 with the multicanonical weight function (right).

two stages:

i) the measurement of the potential barrier (probabilistic suppression) between two integer vacua, and

ii) the calculation of the rate of the tunneling through the top of the potential barrier.

Let us first look at the measurement of the potential barrier. This is the multicanonical stage of the computation. As is usually done in a multicanonical context, instead of 
sampling configurations with the canonical weight

$$
P_{\text {can }}(U, \Phi) \propto e^{-S(U, \Phi)},
$$

we compensate for the strong suppression by adding a carefully-chosen weight function $W\left(N_{\mathrm{CS}}\right)$, which is a function of the order parameter, in this case the Chern-Simons number. The configurations $\{U, \Phi\}$ are now sampled with the probability density

$$
P_{\text {muca }}(U, \Phi) \propto e^{-S(U, \Phi)+W\left(N_{\mathrm{CS}}[U]\right)} .
$$

Defining the physical (canonical) probability distribution of the Chern-Simons number

$$
p_{\text {can }}\left(N_{\mathrm{CS}}^{\prime}\right)=\int \mathrm{d} U \mathrm{~d} \Phi P_{\text {can }}[U, \Phi] \delta\left(N_{\mathrm{CS}}^{\prime}-N_{\mathrm{CS}}[U]\right),
$$

it is clear that the corresponding multicanonical distribution is

$$
p_{\text {muca }}\left(N_{\mathrm{CS}}\right)=p_{\text {can }}\left(N_{\mathrm{CS}}\right) e^{W\left(N_{\mathrm{CS}}\right)} .
$$

Thus, the probability suppression in multicanonical simulations vanishes if we choose $W(N)=-\ln p_{\text {can }}(N)+$ const. This is not a particularly useful result because we do not know the canonical distribution a priori; indeed, that is the quantity we set out to compute with the multicanonical method.

However, it is possible to calculate a good enough approximation for $W$ by using an automatic iterative "self-learning" procedure. Here we follow the method presented in ref. [45], with the obvious modification of using a different order parameter. Essentially, during the learning stage the weight function is continuously modified in order to maximize the flatness of the total distribution of the Chern-Simons number. When the iteration has sufficiently converged, the resulting weight function $W$ is then used in a production run.

The physical (canonical) Chern-Simons probability distribution is now obtained from the measured multicanonical distribution using equation (3.5). An example of the multicanonical evolution and the resulting probability distribution is shown in Figure 4, for $m_{H}=115 \mathrm{GeV}$ and $T=140 \mathrm{GeV}$; the same parameters as the lowest temperature in Figure 3. We recall that in this figure the "evolution" cannot be interpreted as a physical evolution in real time. As we can observe, the distribution of the Chern-Simons number is now almost flat. The resulting canonical (physical) probability distribution is shown in Figure 5, together with the weight function $W\left(N_{\mathrm{CS}}\right)$ used.

We obtain an estimate for the physical expectation value for a general observable $A$ from multicanonical simulation from

$$
\langle A\rangle=\frac{\sum_{i} A_{i} e^{-W_{i}}}{\sum_{i} e^{-W_{i}}},
$$

where the sums go over the configurations $\{U, \Phi\}_{i}$ obtained from the simulation and on which the measurements are performed, $W_{i}=W\left(N_{\mathrm{CS}}\left[\{U\}_{i}\right]\right)$ and $A_{i}=A\left(\{U, \Phi\}_{i}\right)$.

A couple of comments on the practical implementation of the multicanonical sampling using the Chern-Simons number are in order. Because now the Chern-Simons number 
enters in the sampling weight for the configurations, it has to be evaluated in an unbiased manner; that is, the Chern-Simons number for a given gauge field configuration has to be a unique value (modulo 1), independent of the "history" of the configuration. The calibrated cooling described above has a small residual component which depends on the trajectory of the configurations. To ensure unbiased sampling, we do not use the cooled trajectory method but cool down to the vacuum at every measurement of $N_{\mathrm{CS}}$. More precisely, we first cool the gauge field a pre-determined amount (in most cases the cooling time is $\sim 8.4 a^{2}$ ) in order to reduce the UV noise, and then start integrating $\mathbf{E} \cdot \mathbf{B}$ while cooling to vacuum. The frequent cooling now completely dominates the CPU-time budget of the simulation.

The multicanonical probabilistic weight is implemented as an accept/reject step as follows: i) start with configuration $A$, with weight function $W_{A}$; ii) perform one heatbath sweep through the lattice, producing provisional new configuration $B$; iii) measure $N_{\mathrm{CS}}(B)$ as described above, obtaining $W_{B}$. iv) The new configuration is accepted with the probability

$$
p_{\text {accept }}(A \rightarrow B)=\left\{\begin{array}{ll}
1 & \text { if } W_{A} \leq W_{B} \\
e^{W_{B}-W_{A}} & \text { if } W_{A}>W_{B}
\end{array} .\right.
$$

If the update is rejected, we start again at point i) with configuration $A$. The acceptance rate was around $50 \%$ at the lowest temperatures used, and increasing at higher temperatures.

Obviously, the measured value of $N_{\mathrm{CS}}$ depends on the amount of cooling applied before the measurement. Thus, the obtained probability distribution $p\left(N_{\mathrm{CS}}\right)$ is also cooling dependent. However, this is completely cancelled by the dynamical rate measurement described in Section 3.3, so that the final rate is independent of the amount of cooling. Nevertheless, the right amount of cooling must be judiciously chosen for efficiency: insufficient cooling gives too noisy observables, whereas too much cooling takes one too far "downhill" from the original configuration towards the vacuum. In both cases the measured $N_{\mathrm{CS}}$ is too far from the "true" value, and the result is that we do not observe a random walk for $N_{\mathrm{CS}}$. The situation becomes worse at large volumes and coarse lattices.

\subsection{Sphaleron rate in the broken phase: dynamical prefactor}

The multicanonical procedure described above gave us the probability distribution of the Chern-Simons number in the broken phase. We can now measure the tunneling rate following refs. [23, 45]:

1. Let us assume that we have done the multicanonical simulations and obtained the canonical (physical) probability distribution of the Chern-Simons number $p_{\text {phys. }}\left(N_{\mathrm{CS}}\right)$.

2. We choose a narrow interval $1 / 2-\epsilon / 2 \leq N_{\mathrm{CS}} \leq 1 / 2+\epsilon / 2$ around the point that separates vacuum $N_{\mathrm{CS}}=0$ from the vacuum $N_{\mathrm{CS}}=1$. The relative probability of finding a configuration here is

$$
P\left(\left|N_{\mathrm{CS}}-1 / 2\right|<\epsilon / 2\right)=\int_{1 / 2-\epsilon / 2}^{1 / 2+\epsilon / 2} d N p_{\text {phys }}(N) .
$$



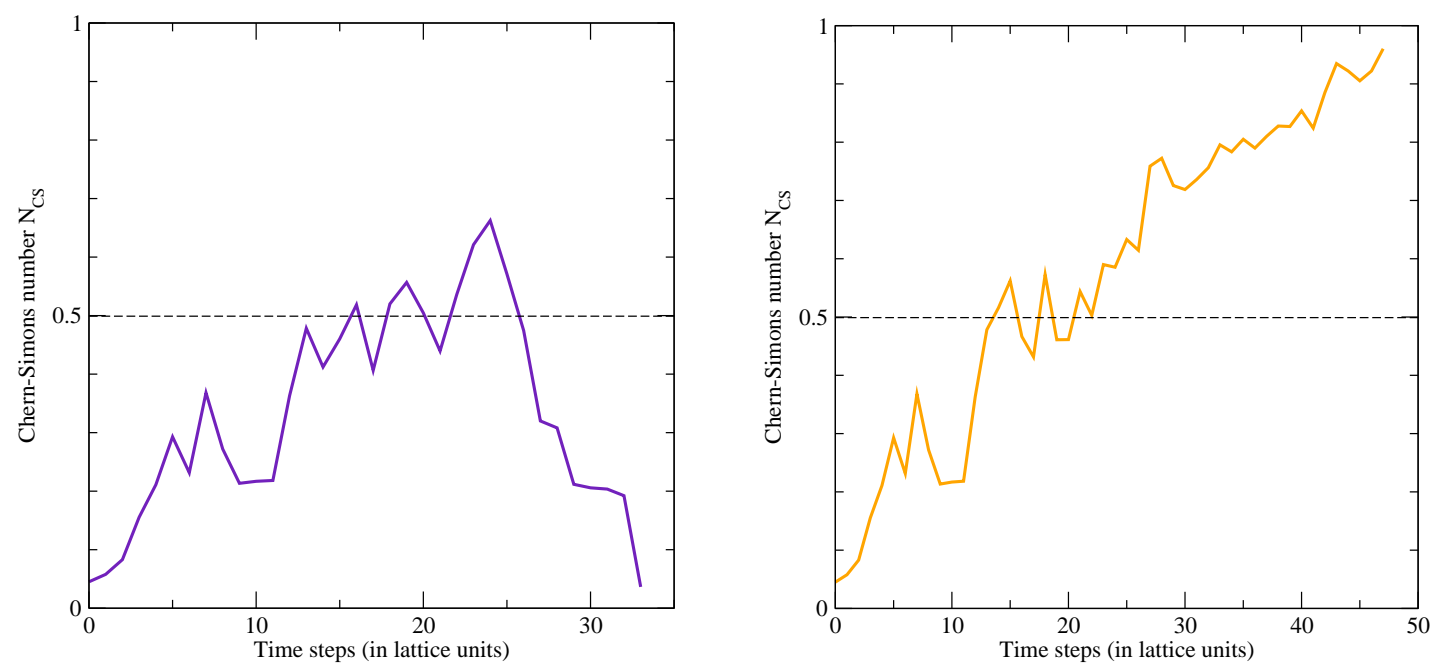

Figure 6. Two real-time trajectories starting from the same configuration. The final and initial configuration can either be the same (left) or different (right). The trajectories cross the central value of our order parameter $N_{\mathrm{CS}}=1 / 2$ several times, a fact we compensate for through the dynamical prefactor (3.9).

This is where we need multicanonical methods, as the probability of being on top of the barrier is extremely small, and to get a reliable estimate would take an impractically long time with canonical sampling.

3. Let us now take a random configuration from the canonical distribution but with the constraint $1 / 2-\epsilon / 2<N_{\mathrm{CS}}<1 / 2+\epsilon / 2$; i.e. near the top of the potential barrier. Starting from this configuration, we now generate two real-time trajectories using the heat-bath dynamics, as described in Section 3.1. The trajectories are evolved until the Chern-Simons number falls near a vacuum value. Interpreting one of the trajectories as evolving backwards in time, we can glue the trajectories together at the starting point and obtain a vacuum-to-vacuum trajectory. The trajectory can either return to the starting vacuum or be a genuine tunneling trajectory, see Figure 6. Only the latter-type trajectories contribute to the sphaleron rate.

4. We can obtain the tunneling rate by measuring $\left|\Delta N_{\mathrm{CS}} / \Delta t\right|$ from the trajectories at the moment they cross the value $N_{\mathrm{CS}}=1 / 2$. Here $\Delta t$ is the time interval between successive measurements, and $\Delta N_{\mathrm{CS}}$ the change in Chern-Simons number. This characterizes the probability flux thorough the top of the barrier. We obtain the physical time difference from the relation between the heat-bath "time" and physical time, equation (2.12).

5. If the tunneling trajectories would go straight across the top, the ingredients above would be sufficient to calculate the total rate. However, typically the trajectories "random walk" near the top of the barrier and can cross the value $N_{\mathrm{CS}}=1 / 2$ several times. Because the trajectories were chosen starting from a set of configurations near the top of the barrier, this leads to overcounting: the evolution could be started at 
any point the $N_{\mathrm{CS}}=1 / 2$ limit is crossed. This can be compensated by calculating a dynamical prefactor

$$
\mathrm{d}=\frac{1}{N_{\text {traj }}} \sum_{\text {traj }} \frac{\delta_{\text {tunnel }}}{\# \text { crossings }},
$$

where the sum goes over the ensemble of trajectories, $N_{\text {traj }}$ is the number of trajectories, $\delta_{\text {tunnel }}$ is 0 if the trajectory does not lead to a change of the vacuum and 1 if it does, and (\# crossings) is the number of times the trajectory crosses $N_{\mathrm{CS}}=1 / 2$.

With these ingredients, the sphaleron rate now becomes

$$
\Gamma=\frac{P\left(\left|N_{\mathrm{CS}}-1 / 2\right|<\epsilon / 2\right)}{\epsilon}\left\langle\left|\frac{\Delta N_{\mathrm{CS}}}{\Delta t}\right|\right\rangle \mathrm{d}
$$

We note that the result is independent of $\epsilon$ as long as $\epsilon \ll 1$. It is also independent of the frequency $\Delta t$ with which the Chern-Simons number is measured: if we decrease the measurement interval, the trajectories become more jagged due to the random-walk nature of the heat-bath updates. This will increase the number of the crossings of the value $N_{\mathrm{CS}}=1 / 2$ and hence decrease d. However, the latter is completely compensated by a corresponding increase in $\left\langle\left|\Delta N_{\mathrm{CS}} / \Delta t\right|\right\rangle$. If the measurement interval $\Delta t$ is small enough, random walk arguments imply $\mathrm{d} \propto(\Delta t)^{1 / 2}$ and $\left\langle\left|\Delta N_{\mathrm{CS}} / \Delta t\right|\right\rangle \propto(\Delta t)^{-1 / 2}$. This is corroborated by the numerical data. Thus, equation (3.10) has a well-defined continuum limit.

\section{Results}

We concentrate on two physically significant observables, the sphaleron rate and the Higgs field expectation value as functions of temperature at $m_{H}=115 \mathrm{GeV}$ and $m_{H}=160 \mathrm{GeV}$. For both quantities we check for the finite volume and finite lattice spacing effects.

\subsection{Higgs field $v(T)$}

The gauge invariant Higgs condensate $\left\langle\phi^{\dagger} \phi\right\rangle$ is a direct probe of the phase transition or cross-over. At high temperatures it is close to zero, and at low temperatures it acquires an expectation value which grows as the temperature decreases. Because of the additive renormalisation, Eq. (2.8), the symmetric-phase value can become negative. The results obtained at $\beta_{G}=9$, lattice size $32^{3}$, are shown in Fig. 7. Note that we display $\left\langle\phi^{2}\right\rangle$ in units of $g^{2} T^{2}$, and goes to infinity as temperature goes to zero.

At high temperature in the symmetric phase, $\left\langle\Phi^{\dagger} \Phi\right\rangle$ is close, but not quite identical, to zero. As the temperature is lowered, we enter the cross-over region where $\left\langle\Phi^{\dagger} \Phi\right\rangle$ grows rapidly. At smaller Higgs masses $\left(m_{H} \lesssim 72 \mathrm{GeV}\right)$, this rapid growth becomes a discontinuous jump, indicating a first-order transition [4]. Below the cross-over region, $\left\langle\Phi^{\dagger} \Phi\right\rangle /\left(g^{2} T^{2}\right)$ settles to an almost linear increase.

For $m_{H}=115 \mathrm{GeV}$ we measured $\Phi^{\dagger} \Phi$ at selected temperature values while varying the lattice spacing by more than a factor of two $\left(\beta_{G}=6 \ldots 16\right)$. The results are shown 

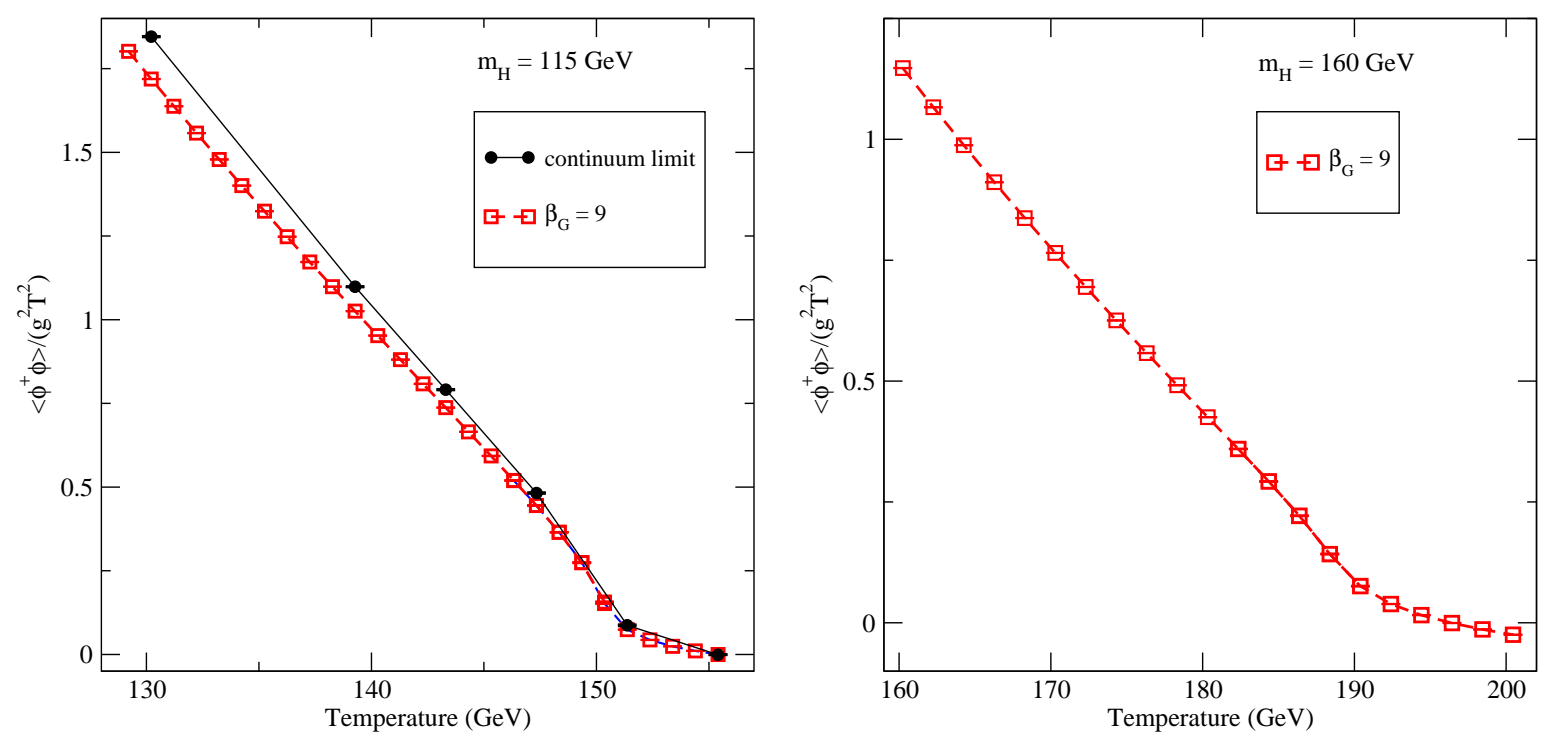

Figure 7. The Higgs field expectation value for $m_{H}=115 \mathrm{GeV}$ (left) and $160 \mathrm{GeV}$ (right). Squares are for $\beta_{G}=9$, volume $(L / a)^{3}=32^{3}$. For $m_{H}=115 \mathrm{GeV}$ we have performed the continuum limit extrapolation at selected temperatures using a range of lattice spacings $\beta_{G}=4 /\left(g_{3}^{2} a\right)=6 \ldots 12$ and extrapolating linearly to continuum. We observe that the $\beta_{G}=9$ result deviates less than $8 \%$ from the continuum limit in the range of temperatures studied. The lines are to guide the eye.

in Figure 8. In this case we can reliably take the continuum limit by linear extrapolation. We observe that when $\left\langle\phi^{\dagger} \phi\right\rangle$ is small, the cut-off effects are very small, and at lowest temperatures $T \approx 130 \mathrm{GeV}$ the $\beta_{G}=9$ result deviates from the continuum limit by less than $8 \%$. The physical volume was kept fixed, at $L g_{3}^{2} \approx 14$. We have checked that this is a large-enough volume so that the residual finite-volume effect is unobservable within our statistical accuracy.

\subsection{The sphaleron rate $\Gamma_{\text {diff }}(T)$}

The sphaleron rate $\Gamma / T^{4}$ for Higgs masses $115 \mathrm{GeV}$ and $160 \mathrm{GeV}$ is shown in Fig. 9, using $\beta_{G}=9$ data. As expected, at high temperature in the symmetric phase, the rate becomes insensitive to the temperature apart from the trivial scaling. In this region the standard "canonical" real-time evolution is sufficient. As we proceed into the cross-over region there is a rather sharp turnover, with a drop of $10^{-3}$ over $5 \mathrm{GeV}$. In this region, both the canonical and multicanonical methods were used, and they agree very convincingly. Deeper in the broken phase, the decrease in the rate flattens out somewhat to a clean exponential dropoff, and, using the multicanonical approach, we were able to follow the rate over 10 orders of magnitude. For comparison, we have included the extrapolation from [26], expected to be valid deep in the broken phase. We see that the slope is correct, but that the central value of the rate is off by about an order of magnitude; or equivalently that the temperature axis is shifted by about $2 \mathrm{GeV}$ for a Higgs mass of $115 \mathrm{GeV}$ and about $5 \mathrm{GeV}$ in the $160 \mathrm{GeV}$ case. The data is shown for $\beta_{G}=9$, where the majority of our simulations were done.

Sphalerons are extended objects, and thus it is necessary to check the finite volume 

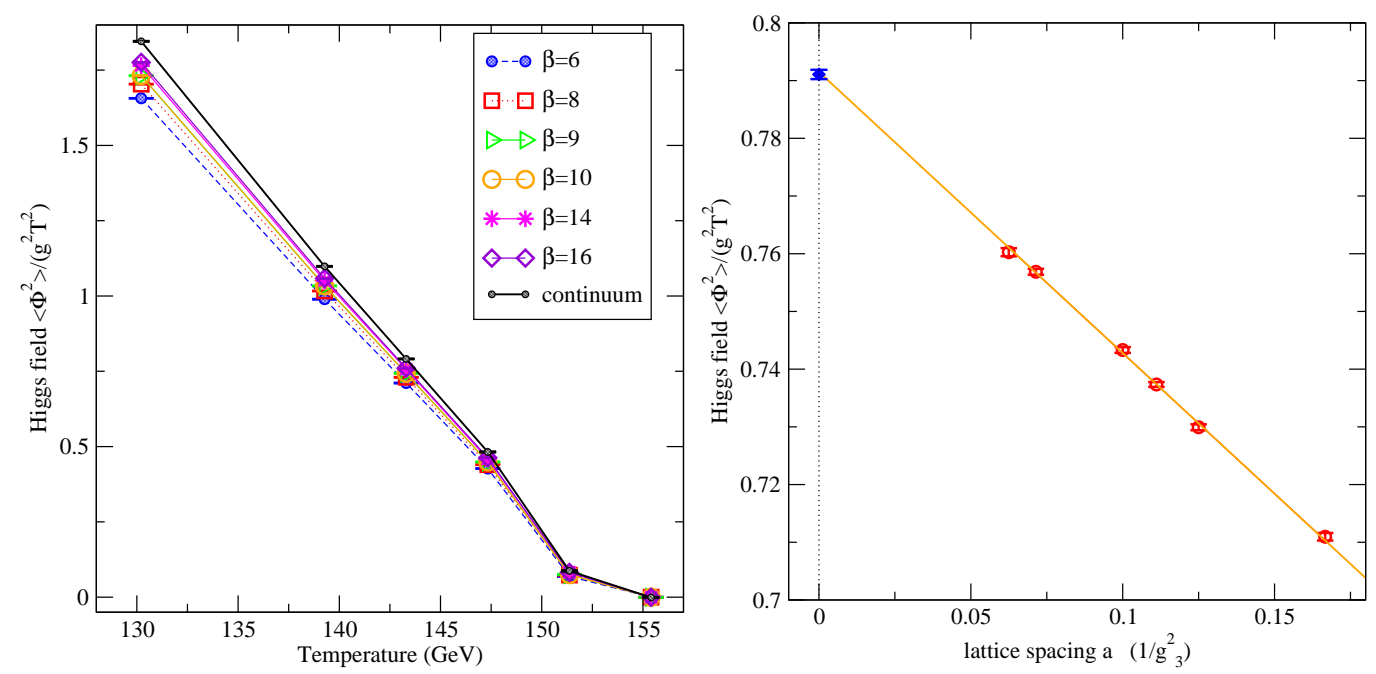

Figure 8. Left: the Higgs field calculated for $m_{H}=115 \mathrm{GeV}$ and several temperatures, with decreasing lattice spacing $a$, but keeping the volume constant, according to Table 2. The black line is the continuum extrapolation. Right: an example of the continuum extrapolation at a single value of the temperature $T=143 \mathrm{GeV}$.
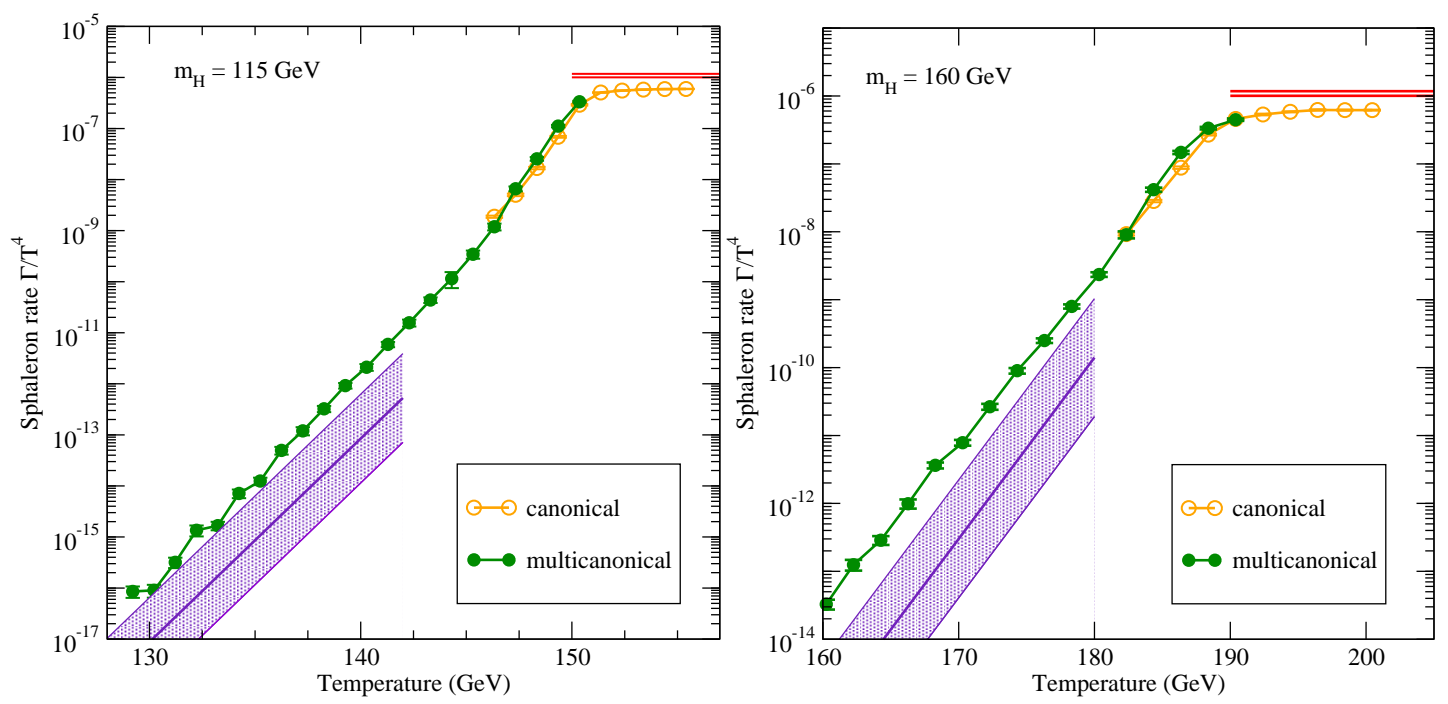

Figure 9. The sphaleron rate for $m_{H}=115 \mathrm{GeV}$ (left) and $160 \mathrm{GeV}$ (right) at $\beta_{G}=9$. The shaded band is the theoretical estimate (plus error ranges extrapolated from lattice results in [23]) for the broken phase and the horizontal lines for the symmetric phase, as calculated in [26]. The canonical and multicanonical results agree within errors at high temperatures.

effects. Using constant lattice spacing $\beta_{G}=9$ and lattice sizes $L / a=16-54$ we observed no systematic finite-size dependence within our statistical accuracy. Thus, we can be confident that $L=32 a \approx 14 / g_{3}^{2}$ is safely large enough at all temperatures. This result is in agreement with ref. [12], where the volume dependence of the sphaleron rate became 
negligible at $L \gtrsim 5 / g_{3}^{2}$ in pure $\mathrm{SU}(2)$ gauge theory.

As we did with the Higgs field expectation value, we investigated the dependence of the sphaleron rate on the lattice spacing. We chose a set of six temperatures in the interval $130-155 \mathrm{GeV}$ and measured the rate at $\beta_{G}=4 /\left(g_{3}^{2} a\right)=6-16$, while keeping the physical volume approximately constant: $L \approx 3.5 \beta_{G}=14 / g_{3}^{2}$. The lattice spacings and volumes are shown in Table 1, and the resulting sphaleron rates are shown in Figure 10.

In the symmetric phase the lattice-spacing dependence is very mild. Deep in the broken phase the rate appears to decrease as $a$ is decreased. This can be understood in the light of the increasing Higgs field expectation value at smaller $a$, see Figure 8.

However, deep in the broken phase $(T \lesssim 145 \mathrm{GeV})$ and for the smallest lattice spacings $\left(\beta_{G} \geq 14\right.$ ) our multicanonical order parameter, cooled $N_{\mathrm{CS}}$ (Figure 2), becomes ineffective and we are not able to obtain a sufficiently accurate measurement of the rate for the proper continuum limit. This is due to the increased noise in the measurement at smaller lattice spacings: when the amplitude of the noise is of order unity, a large fraction of the configurations with (measured) $N_{\mathrm{CS}}$ near half-integer value are actually some distance from the top of the tunneling barrier. Thus, only a small fraction of these configurations will lead to tunneling trajectories.

The amount of noise can be reduced by applying more cooling before the measurement of $N_{\mathrm{CS}}$. However, cooling evolves the configuration towards one of the vacua $\left(N_{\mathrm{CS}}\right.$ integer), and with too much cooling the measured order parameter does not track the true ChernSimons number well enough for effective update. We emphasize that despite these issues the multicanonical method remains exact in the limit of infinite statistics; it is only the efficiency of the method which suffers.

Because of this issue our statistics at small lattice spacing is severely restricted and we cannot obtain a reliable continuum limit. Thus, our final answer remains the $\beta_{G}=9$ result, where we have most of the data. However, what the data indicates is that the true continuum limit is probably a factor of $2-3$ below the $\beta_{G}=9$ result deep in the broken phase, which very likely makes the agreement with ref. [26] in Figure 9 better.

\begin{tabular}{|l|l|l|l|}
\hline$a\left(1 / g_{3}^{2}\right)$ & $\beta_{G}$ & $L$ & $L / \beta_{G}$ \\
\hline 0.67 & 6 & 20 & 3.3 \\
0.5 & 8 & 28 & 3.5 \\
0.44 & 9 & 32 & 3.56 \\
0.4 & 10 & 36 & 3.6 \\
0.29 & 14 & 48 & 3.4 \\
0.25 & 16 & 56 & 3.5 \\
\hline
\end{tabular}

Table 1. Lattice values for the continuum limit. $a$ is the lattice spacing, $\beta_{G}$ is defined in (2.4), $L$ is the size of our volume. From the ratio $L / \beta_{G}$ we notice that we keep the physical proportions constant while we diminish the size of the lattice spacing $a$. 

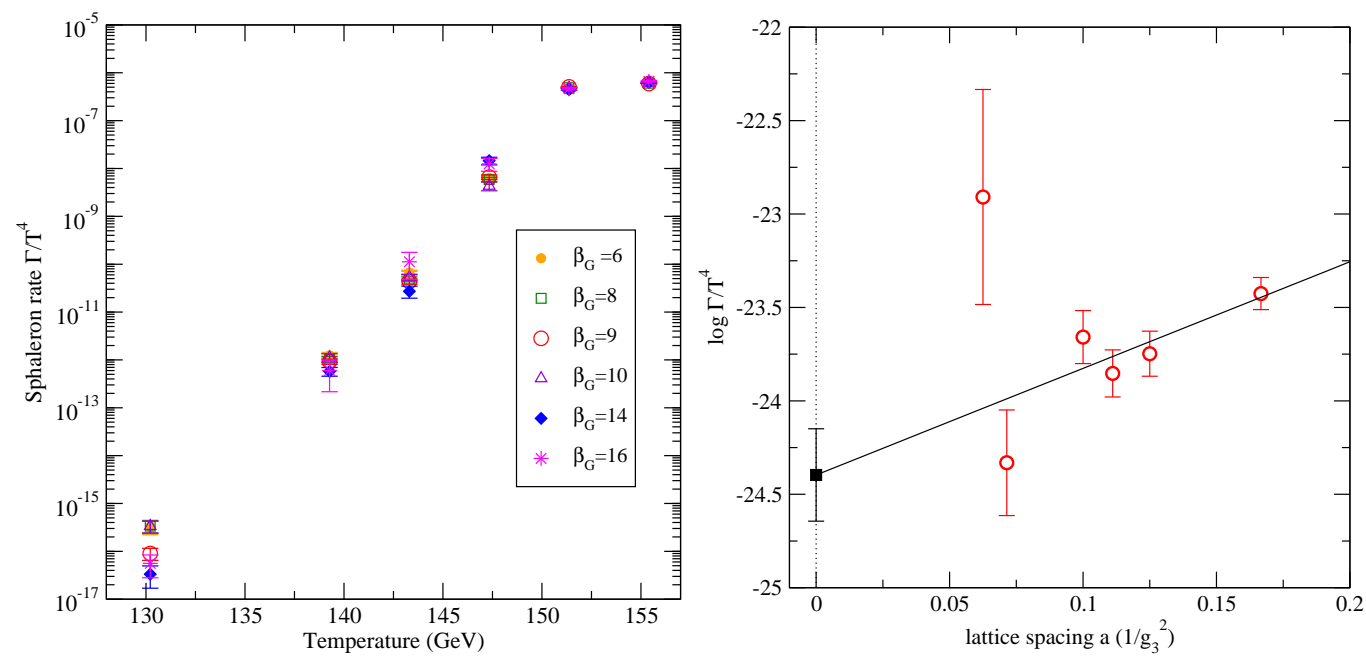

Figure 10. Left: the sphaleron rate calculated for $m_{H}=115 \mathrm{GeV}$ and with different lattice spacings $a$ while keeping the physical volume constant. Right: an example of the rate at $T=143 \mathrm{GeV}$ as a function of the lattice spacing. Here the continuum limit has been extrapolated by assuming that the dominant error of $\log \Gamma$ is linear in $a$. However, if we extrapolate $\Gamma$ itself linearly in $a$ the result is compatible with vanishing rate in the continuum limit.
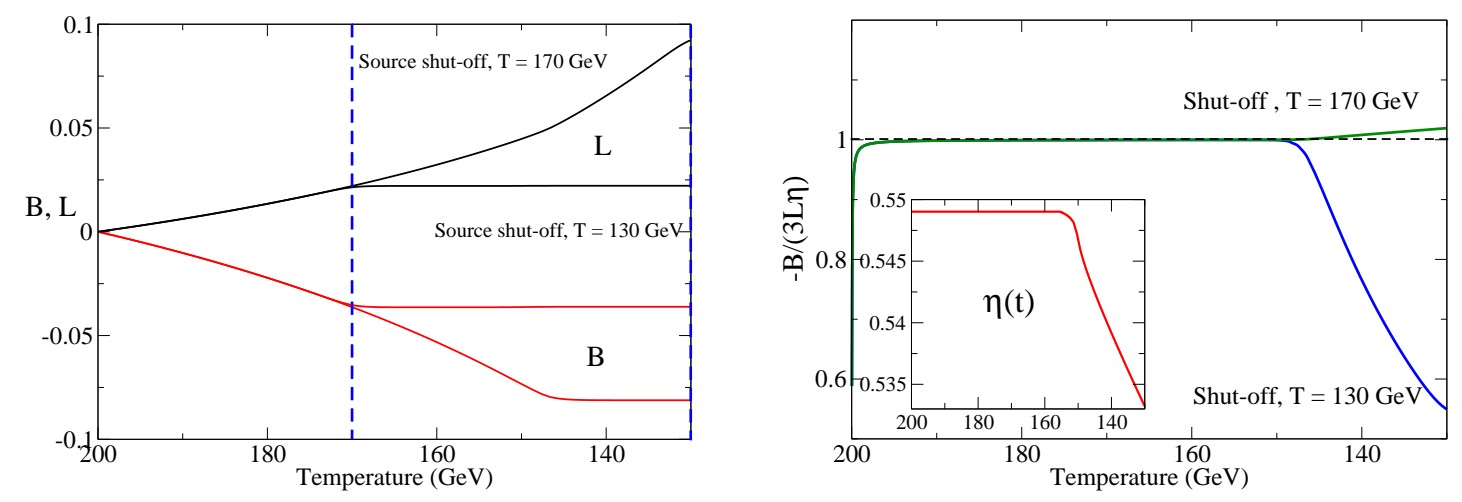

Figure 11. Left: the evolution of baryon number $B$ (red) and lepton number $L$ (black) in the presence of a lepton number source, turned on at $T=200 \mathrm{GeV}$. The source is turned off at $T=170 \mathrm{GeV}$ and $130 \mathrm{GeV}$ respectively (blue dashed lines). Right: The evolution of the ratio (5.8), with the source turned off at $T=170 \mathrm{GeV}$ (green) and $130 \mathrm{GeV}$ (blue). Inserted: the evolution of the quantity $\eta(t)$.

\section{A sample Leptogenesis calculation}

To gauge the impact of using the correct sphaleron rate and Higgs expectation value through the transition, we solved the Leptogenesis equations (1.10)-(1.11), under some simplifying assumptions.

As the sphaleron rate is well-known in the symmetric phase, we focus our interest on 
the cross-over region, to investigate the efficiency of lepton-to-baryon number conversion through the newly-calculated sphaleron rate. In order to enhance the effect of the sphaleron rate suppression at cross-over temperatures, we study two limiting cases: one where the lepton-number source $f_{i}(t)$ was shut off well before the start of the cross-over, and one where we let the source active throughout.

We took $n_{G}=3$ and assumed that all lepton species are equivalent $L_{i}=L, i=1,2,3$, $\sum_{i} L_{i}=3 L$. The initial baryon and lepton numbers vanish $L(t=0)=B(t=0)=0$. The source for the lepton number is therefore taken to be operational between $T=200 \mathrm{GeV}$ and $T=T_{\text {cut }- \text { off }}$,

$$
f_{i}(t)=f(t)=\frac{f_{0}}{2}\left(1-\tanh \left[\frac{T_{\text {cut }- \text { off }}-T}{2 \mathrm{GeV}}\right]\right),
$$

Since the equations are linear, the normalization of $f_{0}$ is arbitrary. This leaves only the source shut-off temperature $T_{\text {cut-off }}$ as a free parameter, which we varied from 170 to $130 \mathrm{GeV}^{4}$, before and after the cross-over, respectively.

The full expressions for $\gamma(t)$ and $\eta(t)$ in (1.10-1.11) read [26]

$$
\gamma(t)=n_{G}^{2} \rho\left(\frac{v(T)}{T}\right)\left[1-\chi\left(\frac{v(T)}{T}\right)\right] \frac{\Gamma_{\text {diff }}(T)}{T^{3}}, \quad \eta(t)=\frac{\chi\left(\frac{v(T)}{T}\right)}{1-\chi\left(\frac{v(T)}{T}\right)},
$$

where $T=T(t)$, and the functions

$$
\begin{aligned}
\rho(x) & =\frac{3\left[65+136 n_{G}+44 n_{G}^{2}+\left(117+72 n_{G}\right) x^{2}\right]}{2 n_{G}\left[30+62 n_{G}+20 n_{G}^{2}+\left(54+33 n_{G}\right) x^{2}\right]}, \\
\chi(x) & =\frac{4\left[5+12 n_{G}+4 n_{G}^{2}+\left(9+6 n_{G}\right) x^{2}\right]}{65+136 n_{G}+44 n_{G}^{2}+\left(117+72 n_{G}\right) x^{2}} .
\end{aligned}
$$

We calculated the evolution of lepton and baryon number from temperature $200 \mathrm{GeV}$ down to $130 \mathrm{GeV}$. Using that to a good approximation in the early Universe $T \propto 1 / a$, with $a(t)$ the scale factor, we have that

$$
\frac{d}{d t}=-H T \frac{d}{d T}
$$

where $H$ is the Hubble rate, given by the (radiation-dominated) Friedman equation ${ }^{5}$

$$
H^{2}=\frac{\pi^{2} g^{*} T^{4}}{90 M_{\mathrm{pl}}^{2}}, \quad g^{*}=106.75, \quad M_{\mathrm{pl}}=2.43 \times 10^{18} \mathrm{GeV} .
$$

Over the range of temperatures used here, $H=(8.8-3.7) \times 10^{-14}$.

Once the source is turned off, and in the limit that $L$ and $B$ evolve much faster than $v$ and $\eta$, we can write the equations in terms of $Y=B+\eta 3 L$

$$
\frac{d \ln Y}{d \ln T}=\frac{\gamma(T)}{H(T)}(1+\eta(t))
$$

\footnotetext{
${ }^{4} \mathrm{We}$ chose the $2 \mathrm{GeV}$ width to mimic a fast shut-off of the source.

${ }^{5}$ We ignored the effect of $g^{*}$ changing slightly as the top quark begins acquiring its mass.
} 
so that $Y=0$ is enforced unless $\gamma / H$ is too small. And so if the sphaleron rate is fast enough, we expect

$$
-\frac{B(T)}{3 L(T) \eta(T)} \simeq 1
$$

We say that the system is in "equilibrium" when this relation is obeyed. We note that $\eta(v(T) / T=0)=0.549 \ldots$ and $\eta(v(T) / T=\infty)=0.48$.

The evolution of $B$ and $L$ in time is shown in Fig. 11 (left). Starting from zero at $T=200 \mathrm{GeV}$, the introduction of the source leads to a growing $L$ and, through sphaleron processes, growing $B$. This continues until the source is switched off; in the examples shown here $T_{\text {cut-off }}=170 \mathrm{GeV}$ and $T_{\text {cut-off }}=130 \mathrm{GeV}$. For the early cut-off, both $B$ and $L$ level off to some asymptotic value. But even without switching off the lepton source, at a temperature around $143 \mathrm{GeV}$ the sphaleron rate becomes inefficient, and the baryon number levels off. Lepton number is still sourced, but having no longer $B$ as a sink, the growth of $L$ becomes steeper. $T_{\text {freeze-out }}=143 \mathrm{GeV}$ corresponds to $\gamma(t) / H \simeq 10$, and $v(T) / T \simeq 0.5$.

Fig. 11 (right) shows the "equilibrium" condition (5.8) in time. We see an initial transient, but from then on the system is nicely in equilibrium until the freeze-out at $T=143 \mathrm{GeV}$. In this case, where the source is still on (blue line), the equilibrium condition is obviously broken by the continued sourcing of $L$. Since $L$ increases and $B$ is constant, the ratio decreases.

What is perhaps more surprising is the ratio (5.8) when there is no source. Because the $v(t)$ increases through the transition, the equilibrium value $\eta(t)$ also changes (see inset). A large enough sphaleron rate relative to the Hubble rate would adjust $B$ relative to $L$ to accommodate this evolving "equilibrium", but as is clear from Fig. 11, this does not happen. $\eta(t)$ just decreases and so the ratio becomes larger than 1. As a consequence, the asymptotic $B$ and $L$ obey

$$
B(x=\infty) \simeq \eta\left(T_{\text {freeze-out }}\right) 3 L\left(T_{\text {freeze-out }}\right)=1.06 \times 3 L\left(T_{\text {freeze-out }}\right) \eta(x=\infty) .
$$

Fig. 11 is based on the $m_{H}=115 \mathrm{GeV}$ data. We did a similar calculation for the $m_{H}=160$ rate, giving the same picture but with $T_{\text {freeze-out }}=175 \mathrm{GeV}$.

\section{Conclusion}

In this paper we have presented the quantitative, non-perturbatively calculated sphaleron rate in the minimal Standard Model, using values of the Higgs mass still (marginally) allowed by experiment, $115 \mathrm{GeV}$ and $160 \mathrm{GeV}$. At these Higgs masses, the electroweak transition is known to be an equilibrium cross-over.

We first probed the temperatures in order to find the range at which the cross-over takes place. We performed lattice simulations in the symmetric phase with canonical Monte Carlo methods using a straightforward heat-bath update algorithm of the Higgs and gauge fields. Here we found that the rate is unsuppressed and follows a random walk in time. When lowering the temperature to reach the broken phase, the rate becomes suppressed 
and in order to observe transitions between vacua we have to switch to multicanonical Monte Carlo and real-time simulations.

We see a perfect match between the data points obtained with canonical and multicanonical Monte Carlo, with a smooth overlap in the cross-over region. This gives us a cross-check on the validity of the results. And by varying the physical volume and the lattice spacing, we found that both the sphaleron rate and Higgs field are close to their infinite-volume and continuum limits.

We obtained the sphaleron rate as a function of the temperature, showing an obvious similarity in the rate at the two Higgs masses $m_{H}=115 \mathrm{GeV}$ and $160 \mathrm{GeV}$, Figure 9 . In both cases, the rates become exponentially suppressed in the broken phase with similar slopes, while having the same $\left(\sim 5 \times 10^{-7} \mathrm{~T}^{4}\right)$ asymptotic value in the symmetric phase. We point out that the cross-over range is clearly related to the Higgs mass: $130-147 \mathrm{GeV}$ for $m_{H}=115 \mathrm{GeV}$, and $160-182 \mathrm{GeV}$ for $m_{H}=160 \mathrm{GeV}$. The cross-over range is clearly noticeable also in the plots for the Higgs field.

Moreover, right at the beginning of the cross-over, the sphaleron rate drops at its fastest. Both in the plots for the sphaleron rate and for the Higgs field, the curves become steepest promptly after the cross-over kicks in, around $145-148 \mathrm{GeV}$ for the smaller Higgs mass and $177-185 \mathrm{GeV}$ for the bigger one.

The behaviour of the sphaleron rate we found is in agreement with results quoted in the literature, in the range where they exist. Our asymptotic value is of the same order of magnitude as in [26]. Direct comparison in the broken phase shows that while the slope is the same, the value is off by an order of magnitude.

Finally, we input the obtained sphaleron rate into a simple-minded model of Leptogenesis. We found that although the rate cuts off exponentially at the transition, the freeze-out of the baryon and lepton number happens slightly later, when the rate is about 10 in units of the Hubble rate. For precision calculations of the generated baryon asymmetry in such models, it is therefore important to take the gradual shut-off of sphaleron processes into account.

\section{Acknowledgments}

This work is supported by the Academy of Finland grants 114371 and 1134018 . The computations have been made at the Finnish IT Center for Science (CSC), Espoo, Finland. M. D. acknowledges support from the Magnus Ehrnrooth foundation. A. T. is supported by the Carlsberg Foundation.

\section{References}

[1] V. A. Kuzmin, V. A. Rubakov and M. E. Shaposhnikov, Phys. Lett. B 155 (1985) 36.

[2] V. A. Rubakov and M. E. Shaposhnikov, Usp. Fiz. Nauk 166 (1996) 493 [Phys. Usp. 39 (1996) 461] [arXiv:hep-ph/9603208].

[3] G. 't Hooft, Phys. Rev. D 14 (1976) 3432 [Erratum-ibid. D 18 (1978) 2199]. 
[4] K. Kajantie, M. Laine, K. Rummukainen and M. E. Shaposhnikov, Phys. Rev. Lett. 77 (1996) 2887 [arXiv:hep-ph/9605288].

[5] K. Kajantie, M. Laine, K. Rummukainen and M. E. Shaposhnikov, Nucl. Phys. B466 (1996) 189-258. [hep-lat/9510020].

[6] K. Rummukainen, M. Tsypin, K. Kajantie, M. Laine and M. E. Shaposhnikov, Nucl. Phys. B 532 (1998) 283 [hep-lat/9805013].

[7] F. Csikor, Z. Fodor and J. Heitger, Phys. Rev. Lett. 82, 21 (1999) [hep-ph/9809291]; Y. Aoki, F. Csikor, Z. Fodor and A. Ukawa, Phys. Rev. D60, 013001 (1999) [hep-lat/9901021].

[8] F. R. Klinkhamer and N. S. Manton, Phys. Rev. D 30, 2212 (1984).

[9] O. Philipsen, Phys. Lett. B 358, 210 (1995) [arXiv:hep-ph/9506478].

[10] J. Ambjorn and A. Krasnitz, Phys. Lett. B 362, 97 (1995) [arXiv:hep-ph/9508202].

[11] J. Ambjorn and A. Krasnitz, Nucl. Phys. B 506, 387 (1997) [arXiv:hep-ph/9705380].

[12] G. D. Moore and K. Rummukainen, Phys. Rev. D 61, 105008 (2000) [arXiv:hep-ph/9906259].

[13] P. B. Arnold, D. Son and L. G. Yaffe, Phys. Rev. D 55, 6264 (1997) [arXiv:hep-ph/9609481].

[14] P. B. Arnold, Phys. Rev. D 55 (1997) 7781 [arXiv:hep-ph/9701393].

[15] G. D. Moore, C. r. Hu and B. Muller, Phys. Rev. D 58, 045001 (1998) [arXiv:hep-ph/9710436].

[16] D. Bodeker, G. D. Moore and K. Rummukainen, Phys. Rev. D 61 (2000) 056003 [arXiv:hep-ph/9907545].

[17] D. Bodeker, Phys. Lett. B 426 (1998) 351 [arXiv:hep-ph/9801430].

[18] G. D. Moore, Nucl. Phys. B 568, 367 (2000) [arXiv:hep-ph/9810313].

[19] P. B. Arnold and L. G. Yaffe, Phys. Rev. D 52 (1995) 7208 [arXiv:hep-ph/9508280].

[20] P. B. Arnold and L. G. Yaffe, Phys. Rev. D 62 (2000) 125014 [arXiv:hep-ph/9912306].

[21] W. H. Tang and J. Smit, Nucl. Phys. B 482 (1996) 265 [arXiv:hep-lat/9605016].

[22] G. D. Moore, Phys. Rev. D 62, 085011 (2000) [arXiv:hep-ph/0001216].

[23] G. D. Moore, Phys. Rev. D 59, 014503 (1999) [arXiv:hep-ph/9805264].

[24] G. D. Moore, Phys. Lett. B 439, 357 (1998) [arXiv:hep-ph/9801204].

[25] H. P. Shanahan and A. C. Davis, Phys. Lett. B 431 (1998) 135 [arXiv:hep-ph/9804203].

[26] Y. Burnier, M. Laine and M. Shaposhnikov, JCAP 0602 (2006) 007 [arXiv:hep-ph/0511246].

[27] C. Amsler et al. [Particle Data Group], Phys. Lett. B 667 (2008) 1.

[28] A. G. Cohen, D. B. Kaplan and A. E. Nelson, Ann. Rev. Nucl. Part. Sci. 43 (1993) 27 [arXiv:hep-ph/9302210].

[29] M. Fukugita and T. Yanagida, Phys. Lett. B 174 (1986) 45.

[30] M. A. Luty, Phys. Rev. D 45 (1992) 455.

[31] A. D. Linde, Phys. Lett. B96 (1980) 289.

[32] K. Kajantie, M. Laine, K. Rummukainen and M. E. Shaposhnikov, Nucl. Phys. B458 (1996) 90-136. [hep-ph/9508379]. 
[33] K. Farakos, K. Kajantie, K. Rummukainen and M. E. Shaposhnikov, Nucl. Phys. B425 (1994) 67-109. [hep-ph/9404201].

[34] K. Farakos, K. Kajantie, K. Rummukainen and M. E. Shaposhnikov, Nucl. Phys. B442 (1995) 317-363. [hep-lat/9412091].

[35] P. H. Ginsparg, Nucl. Phys. B 170 (1980) 388.

[36] T. Appelquist and R. D. Pisarski, Phys. Rev. D23 (1981) 2305.

[37] S. Nadkarni, Phys. Rev. D27 (1983) 917.

[38] N. P. Landsman, Nucl. Phys. B322 (1989) 498.

[39] A. Jakovac, K. Kajantie and A. Patkos, Phys. Rev. D49 (1994) 6810-6821. [hep-ph/9312355].

[40] E. Braaten and A. Nieto, Phys. Rev. D 51 (1995) 6990 [hep-ph/9501375].

[41] M. Laine, Nucl. Phys. B451 (1995) 484-504. [hep-lat/9504001].

[42] G. D. Moore, Nucl. Phys. B523 (1998) 569-593. [hep-lat/9709053].

[43] D. Bödeker, L. McLerran and A. Smilga, Phys. Rev. D52, 4675 (1995) [hep-th/9504123]. Phys. Rev. D55, 6264 (1997) [hep-ph/9609481].

[44] E. Braaten and R. Pisarski, Nucl. Phys. B337, 569 (1990); J. Frenkel and J. Taylor, Nucl. Phys. B334, 199 (1990); J. Taylor and S. Wong, Nucl. Phys. B346, 115 (1990); J. Frenkel and J. Taylor, Nucl. Phys. B 374, 156 (1992); E. Braaten and R. Pisarski, Phys. Rev. D 45, 1827 (1992); J. P. Blaizot and E. Iancu, Phys. Rev. Lett. 70, 3376 (1993) [hep-ph/9301236]; Nucl. Phys. B417, 608 (1994) [hep-ph/9306294]; V.P. Nair, Phys. Rev. D48, 3432 (1993) [hep-ph/9307326].

[45] G. D. Moore and K. Rummukainen, Phys. Rev. D63 (2001) 045002. [hep-ph/0009132]. 